\title{
ATTENUATION OF GRAVITY WAVES BY TURBULENCE
}

\author{
Jose Beya $^{1}$, William Peirson ${ }^{2}$ and Michael Banner ${ }^{3}$
}

\begin{abstract}
We report new laboratory measurements of the interaction between mechanically-generated gravity waves and turbulence generated by simulated rain. Wave attenuation coefficients and vertical profiles of turbulent velocity fluctuations were measured. Observations are in broad agreement with Teixeira and Belcher (2002) despite substantial differences between assumed and measured turbulence profiles. Wave attenuation due to surface turbulence appears to be stronger than theoretical estimates. These finding could have significant implications for the next generation of spectral wave models and the understanding of wave dissipation processes.
\end{abstract}

Keywords: wave-turbulence interaction; wave attenuation; turbulence measurement, wave modelling.

\section{INTRODUCTION}

The co-existence of waves and turbulence is a common phenomenon in the ocean, particularly during storms when waves and turbulence interact in an extremely intricate manner. Our present understanding of wave-turbulence interactions is relatively weak. Correct understanding of intense waves, surges and currents and their impact on environmental, engineering and navigational assets has significant implications for the assessment of the potential for human life and material losses during severe storms.

Present calculation methods provide poor estimates of the hydrodynamics under these extreme conditions. Engineering designs rely on parameterizations based on empirical laboratory data that incorporate such interactions implicitly.

In spite of remarkable improvements wave modelling has experienced in recent years, uncertainties in wave generation and dissipation parameterisations are still of major concern. Model reliability depends strongly on the adequate calibration of the parameters in the generation and dissipation terms. Research efforts in this area have not yet delivered a good understanding of these two processes fundamentally due to the complexity of the wave-turbulence interaction.

Theoretical estimates of wave attenuation due to turbulence validated with limited field and experimental data (Teixeira and Belcher 2002, Ardhuin and Jenkins 2006) have recently been incorporated in wave models, e.g. WAVEWATCH III (Tolman 2009).

Thus, observations providing new understanding on wave-turbulence interactions are fundamental for adequately representing hydrodynamic and associated transport processes in the sea.

In this contribution we report new laboratory measurements of the attenuation of mechanicallygenerated gravity waves and turbulence generated by simulated rain. Turbulence induced by rainfall is produced at the water surface and is depth decaying as observed in the ocean under wind and whitecapping conditions (Craig and Banner 1994). Wave attenuation and turbulence profiles were measured and compared with existing wave-turbulence interaction theories.

\section{EXPERIMENTAL FACILITIES}

Experiments were carried out in a $30 \mathrm{~m}$ long, $0.6 \mathrm{~m}$ wide and $0.6 \mathrm{~m}$ total depth wave tank with glass sidewalls (Figure 1). A programmable servo-controlled actuator drives a flexible plate wave generator cantilevered at near the tank floor. For this study, only monochromatic waves were generated. Waves were absorbed at the end of the tank by a gently-sloping beach. The mean water depth was maintained at $0.415 \mathrm{~m} \pm 1 \mathrm{~mm}$ for all experiments by an automatic control system. Over the measurement period, the water temperature varied from 10.9 to $12^{\circ} \mathrm{C}$.

Vertical artificial rain was generated with a simulator physically identical of that developed by (Shelton et al. 1985) which was designed and shown to produce near uniform droplet fields with size distributions similar to natural rainfall at terminal vertical velocities across a range of rain intensities from 85 to $168 \mathrm{~mm} \mathrm{~h}^{-1}$. Eight 30WSQ nozzles were installed at $3.00 \mathrm{~m}$ above the tank surface located with a spacing of $2.13 \mathrm{~m}$ along the tank. Separate water and air manifold systems supplied pressurised air and water immediately upstream of the nozzles, the compressed air increasing the exit velocity of

\footnotetext{
${ }^{1}$ Water Research Laboratory, University of New South Wales, 110 King St. Manly Vale 2093, NSW, Australia

${ }^{2}$ Water Research Laboratory, University of New South Wales, 110 King St. Manly Vale 2093, NSW, Australia

${ }^{3}$ School of Mathematics, University of New South Wales, Kensington, 2052, NSW, Australia
} 
the water droplets. Water from the tank was recirculated through the rainfall system. The mean rainfall rate was controlled by a rotameter.

During this investigation, measurements were undertaken for two rainfall conditions: a so-called low rainfall (LR) of $108 \pm 7 \mathrm{~mm} \mathrm{~h}^{-1}$ and a high rainfall (HR) of $141 \pm 6 \mathrm{~mm} \mathrm{~h}^{-1}$. These conditions were achieved by setting the flow rates and nozzle air pressures in accordance with the corresponding values in Table 2 of (Shelton et al. 1985). Rainfall intensity and uniformity was confirmed during the experiments by visual inspection and measurements using temporary rain gauges located beneath each nozzle.

Any slicks on the surface of the tank were carefully visually monitored and, prior to testing each day, removed by generating steep waves for approximately 1 hour which carried any surface material to the downstream end of the tank by the Stokes drift. A fan was used to ensure that surface slick material was swept to and retained on the beach (Figure 1).

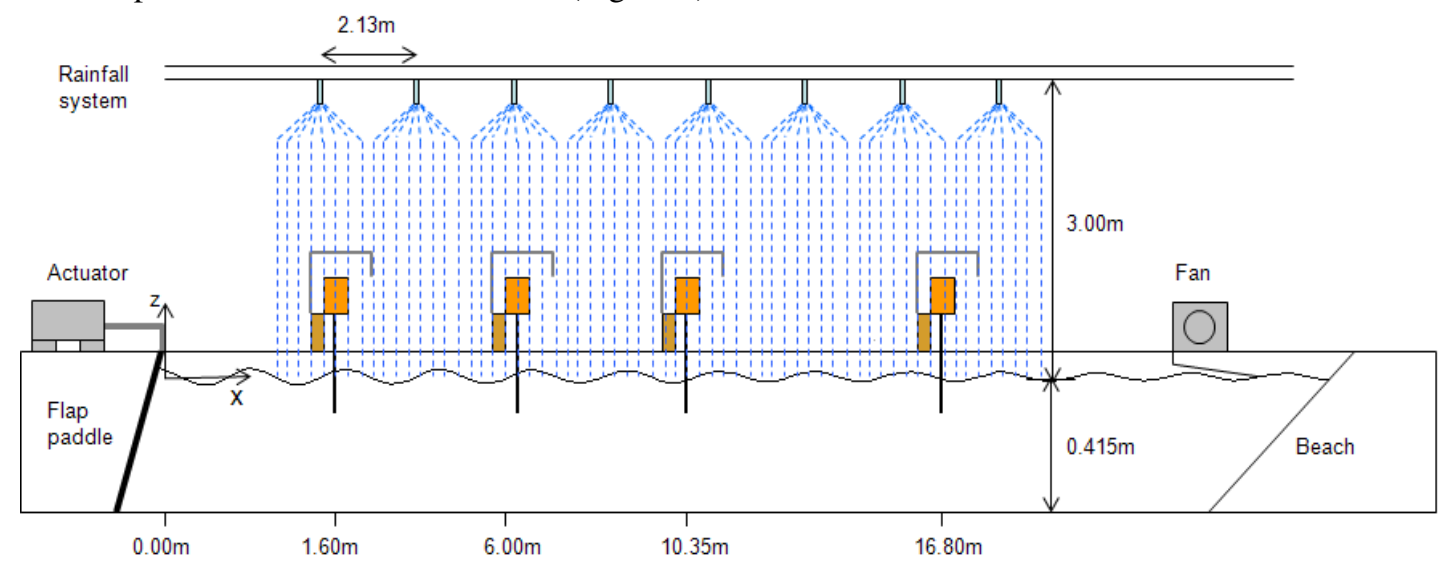

Figure 1. Schematic diagram showing experimental layout and key equipment (not to scale).

\section{Turbulence measurements}

The near-surface velocity field generated by the rain was measured in the absence of any mechanically-generated waves using a Sontek A827 side-looking $16 \mathrm{MHz}, 5 \mathrm{~cm}$ focal distance, threedimensional micro acoustic Doppler velocimeter (ADV). The ADV was used to measure the vertical profile of the turbulent velocity fluctuations near the water surface under rainfall. The ADV was mounted on a static structure with a system that allowed the head to move vertically but with the ADV measurement volume projecting away from its body and any supporting appurtenances. It was important to ensure that the turbulence measurements were taken beneath a sufficiently clear area of surface freely irradiated with rain droplets.

The water column was seeded with $10-30 \mu \mathrm{m}$ diameter white pliolite and rendering clay which was then mixed over the entire depth at least 5 minutes before the start of data recording. The seeding was required to maintain an acoustic signal to noise ratio greater than 15 during the measurement period and the time delay before recording was to allow turbulence generated by the stirring to be dissipated. Testing showed that 5 minutes was an adequate delay to ensure that the measurements were not contaminated by the initial seeding process. An ADV beam check was also carried out before each measurement.

Preliminary static measurements showed that rainfall generated turbulent velocities were very small. Consequently, for static measurements the ADV velocity range was set at its most sensitive level of $\pm 30 \mathrm{~mm} \mathrm{~s}^{-1}$. Measurement ensembles consisting of $163.84 \mathrm{~s}$ of $25 \mathrm{~Hz}$ velocity samples were used to characterise the turbulence over a depth range between $0.031 \mathrm{~m}$ and $0.151 \mathrm{~m}$.

In an attempt to measure the turbulent dissipation rate the ADV was mounted on a trolley that could move at a constant speed ( $U_{\text {profile }}=0.085 \mathrm{~m} \mathrm{~s}^{-1}$ ) much greater than the turbulent fluctuations $\left(u^{\prime}\right)$. This was required to satisfy the Taylor's frozen turbulence hypothesis as no advective mean flow was present in the tank (Tennekes and Lumley, 1974, p. 253). Measurements were taken moving the trolley in the negative $x$ direction with the ADV set as for the static measurements (Figure 1). Test conditions were similar to those for the static measurement except that the ADV velocity range setting had to be increased to $\pm 0.3 \mathrm{~m} \mathrm{~s}^{-1}$. 


\section{Wave measurements}

Four pairs of capacitance probes were located along the tank at fetches $1.60 \mathrm{~m}, 6.00 \mathrm{~m}, 10.35 \mathrm{~m}$ and $16.80 \mathrm{~m}$ from the wave paddle to measure wave attenuation (Figure 1). Each probe was calibrated at least twice before and after measurements and showed gain stability better than $\pm 2 \%$. Wave development along the tank was monitored by capturing $102.4 \mathrm{~s}$ of data at a $600 \mathrm{~Hz}$ sample rate per channel using a National Instruments PCI-6225 data acquisition card fitted to a conventional personal computer. The capacitance wave probe noise levels at the sampling rate for static conditions had a standard deviation less than $0.05 \mathrm{~mm}$.

The attenuation of monochromatic waves with frequencies between 10.5 and $21.0 \mathrm{rad} \mathrm{s}^{-1}$ and mean steepnesses $(a k)$ from 0.05 to 0.15 were measured during the course of the investigation. The ceiling value of $a k=0.15$ was determined from preliminary observations of the formation of Benjamin-Feir (1967) instabilities in the absence of rainfall. At steepnesses greater than 0.15 , wave trains were found to degenerate into groups sufficiently steep to initiate breaking within the test section. Breaking would directly remove energy from the wave field and would contaminate the measurement approach taken during this study.

For each test case, wave generation commenced at least two minutes prior to data collection. Initial measurements were undertaken with no rain (denoted NR hereafter) to obtain the background viscous attenuation within the test facility.

\section{DATA PROCESSING}

\section{Velocity fluctuations}

Representative velocity spectra obtained from the static ADV measurements are shown in Figure 2. Following (Voulgaris and Trowbridge 1997) and (Nikora and Goring 1998), the ambient acoustic noise was determined directly from the measured velocity spectra. In Figure 2, the instrument acoustic noise level is clearly apparent above $45 \mathrm{rad} \mathrm{s}^{-1}$.

The spectra shown in Figure 2 also show low frequency $\left(<8 \mathrm{rad} \mathrm{s}^{-1}\right)$ velocity fluctuations induced by seiches and other low frequency motions within the tank itself. The intensities of velocity fluctuations directly induced by the rain were calculated by partitioning the spectra at the minimum spectral level at the lower frequencies and then deducting the acoustic noise from the remaining high frequency spectrum. This process assumes that the instrument noise is uncorrelated to the velocity fluctuations (Bradshaw 1971).

In agreement with the manufacturer's specifications the noise level was approximately 5 times greater for those velocity components measured parallel to the ADV head ( $v^{\prime}$ and $w^{\prime}$ in this present study, Figure 1) in comparison to the head-normal component ( $u$ '). From Figure 2 the $u^{\prime}$ component shows a significant lower noise level while the similarity between the $w$ ' and $v^{\prime}$ components shows the isotropic nature of the turbulence. Thus, we considered appropriate to assume that the vertical velocity fluctuations profile is isotropic and it is most accurately represented by the measured $u$ ' ADV component. 


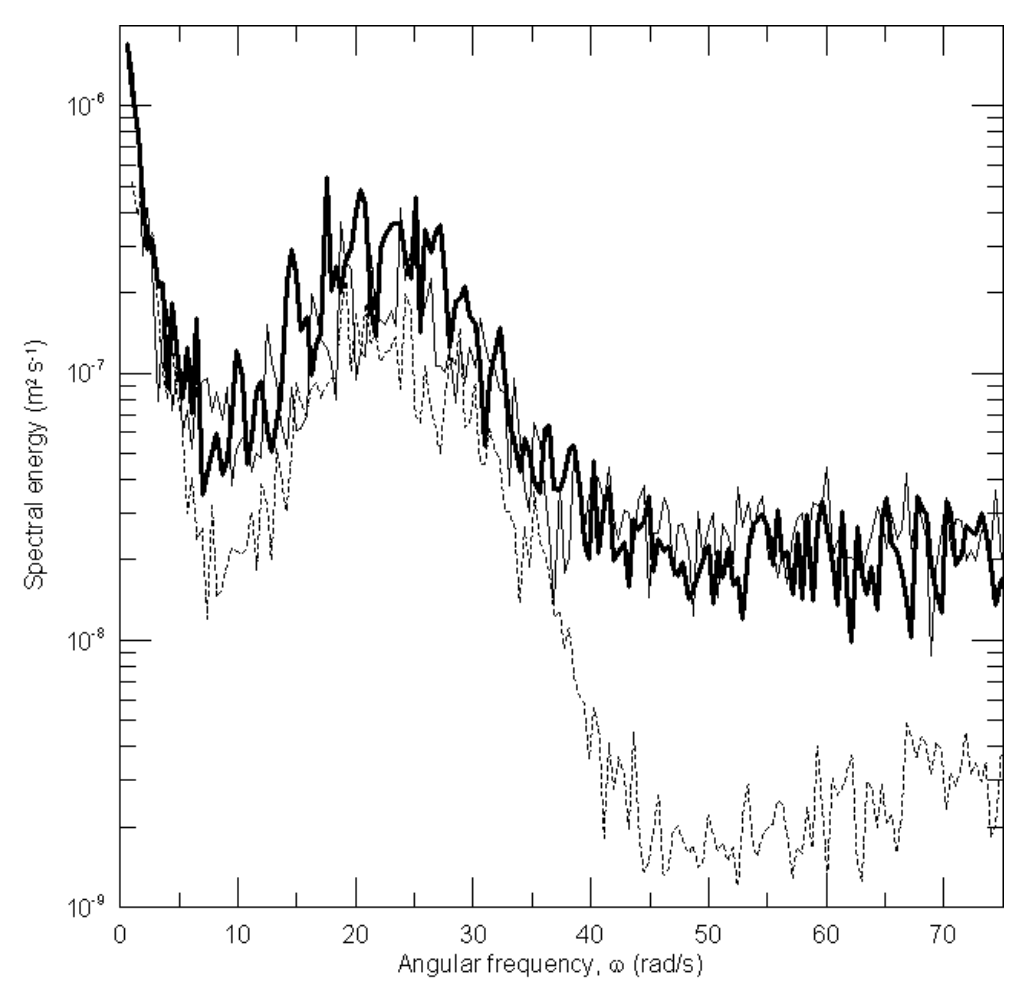

Figure 2. Representative fluctuating velocity spectra obtained from the acoustic Doppler velocimeter in static mode, $u$ ' light dashed line, $v^{\prime}$ heavy solid line, $w^{\prime}$ light solid line. Note the clearly defined minimum in spectral energy at approximately $8 \mathrm{rad} \mathrm{s}^{-1}$ and approximately constant acoustic noise level above $45 \mathrm{rad} \mathrm{s}^{-1}$. Note also the much lower acoustic noise of the head-normal velocity component and the similarity between the $w^{\prime}$ and $v$ ' component showing isotropic turbulence.

\section{Integral turbulence length}

Measurements of turbulent dissipation rates are conventionally obtained by fitting the Kolmogorov model of the sub inertial range of the wave number spectrum under the assumption of isotropic turbulence where the sub inertial range is given by:

$$
\Phi_{k}=C_{1}{ }^{\prime} \varepsilon^{2 / 3} k^{-5 / 3}
$$

where $\Phi_{\mathrm{k}}=$ wave number spectra of the velocity fluctuations, $C_{l}{ }^{\prime}=0.65, \varepsilon=$ turbulent kinetic energy dissipation rate per unit volume and $k=$ characteristic eddy wave number, (Pope 2000, p. 232).

The wave number spectrum can be obtained from the moving trolley ADV measurements using the approximation $\left(x=t \cdot U_{\text {profile }}\right)$ from the Taylor's frozen turbulence hypothesis (Figure 3 ).

For each depth a smoothed spectrum was obtained averaging four repeat measurements. The corresponding wave number spectrum in the presence of rainfall (with acoustic noise deducted) yielded an energy peak at the integral turbulence length $(l)$ and a form of energy spectrum compatible with determining a dissipation rate. However we decided not to report the dissipation measurements due to:

1) The high level of noise in these measurements induced by the increased ADV velocity range and the vibrations in the lowest noise component ( $x$ direction) induced by the movement of the trolley.

2) The weakness of the turbulence (small turbulence Reynolds, $R e_{\lambda}<15$ ). Kolmogorov's theory was developed for steady isotropic turbulence with a well developed sub-inertial range and high $\operatorname{Re}_{\lambda}$ (Pope 2000).

Despite the difficulty in obtaining accurate estimates of the dissipation rate, we observed that the integral turbulence length $(l)$ given by the peak of the spectrum was not severely affected by the noise. The vertical profile of $l$ was obtained from the $w$ ' wave number spectrum which exhibited the lowest noise level and an approximately white spectral response in the absence of rainfall (Figure 3 ). The error in $l$ was estimated from the upper and lower wave number values at the peak of the spectrum. 


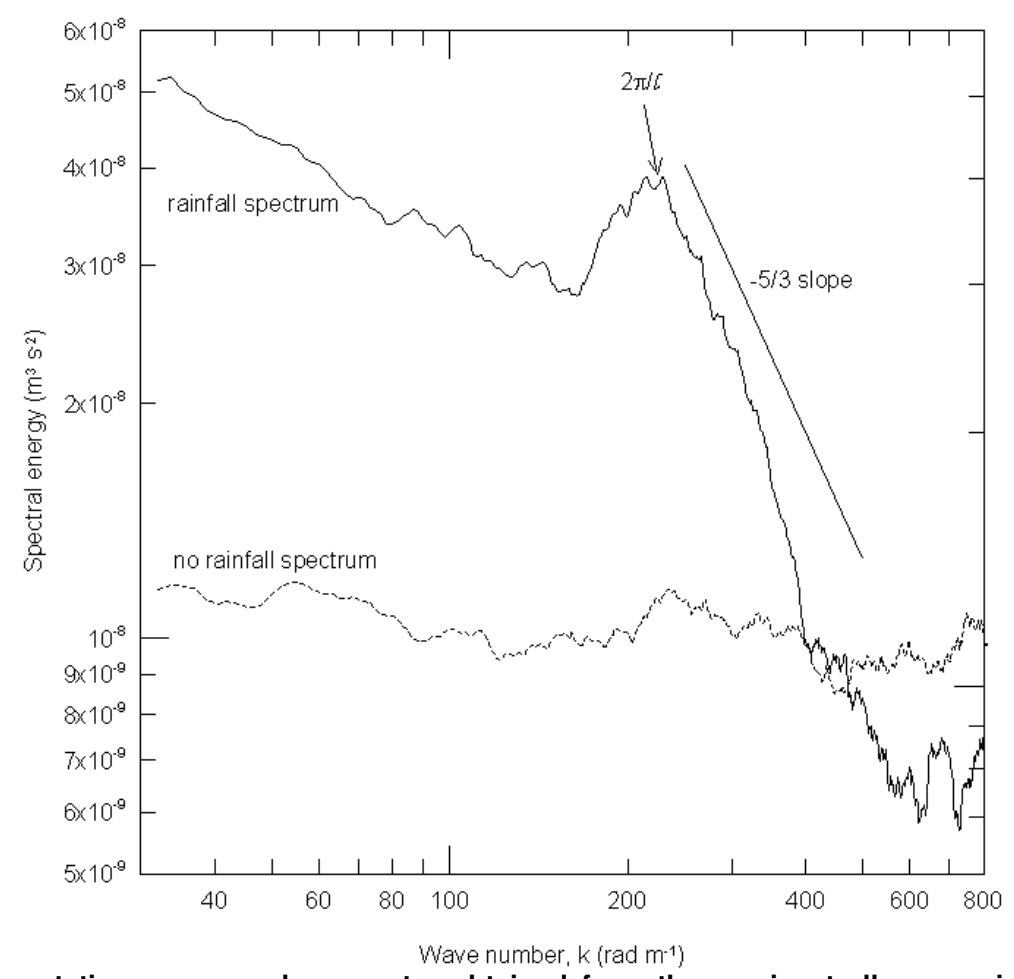

Figure 3. Representative wave number spectra obtained from the moving trolley experiments for the w' velocity component (ADV mounted at $0.037 \mathrm{~m}$ depth). Plotted spectra are the mean of 4 independent measurements, smoothed with 11 point bin averages. Dashed line shows the spectrum obtained in the absence of rainfall and shows little modulation with wave number. Solid line shows the high rainfall case (HR). A line with $-5 / 3$ slope is shown as a reference. The integral length scale (I) for the high rainfall case is indicated. Note the higher noise levels in comparison with the static measurements shown in Figure 2.

\section{Wave attenuation}

Water elevation data sampled at $600 \mathrm{~Hz}$ was averaged using 15 point bins to obtain a filtered $40 \mathrm{~Hz}$ signal prior to spectral processing. A representative set of spectra obtained for the three rain conditions investigated in this study are shown in Figure 4. Fast Fourier transform techniques were used to compute the energy characteristic of the monochromatic waves from each water level time series. The high digitisation rate coupled with the large FFT sample size enabled excellent resolution and extraction of the monochromatic wave energy. As shown in Figure 4, the spectral energy of the monochromatic waves can be clearly distinguished from the gravity-capillary energy of waves generated by the rain. In the absence of rain, the non-linear harmonics of the fundamental wave are clearly apparent in these spectra highlighting the low noise characteristics of the wave probes. The energy associated with a monochromatic wave of angular frequency $\omega_{p}$ was extracted from each record by integrating the spectral energy within the angular frequency band $(1 \pm 0.05) \omega_{p}$.

Wave attenuation was assumed to be exponential and of identical form to that used by Peirson et al (2003):

$$
E=E_{0} e^{-\Delta_{T} x}
$$

where $x$ is fetch along the tank, $E$ is the local wave energy relative to a reference level $E_{0}$ and $\Delta_{T}$ is the total spatial damping coefficient.

Figure 5 shows the decline in monochromatic wave energy with fetch for 15.7 rad.s $\mathrm{s}^{-1}$ angular frequency waves and different steepnesses under the action of rainfall. For each experiment $\Delta_{T}$ was determined by least-squares fit of the data and $90 \%$ confidence limits determined according to the method described by Peirson et al (2003), p.354. The correlation coefficient systematically decreased with decreasing wave frequency reflecting the low attenuation rates at the lower frequencies. 


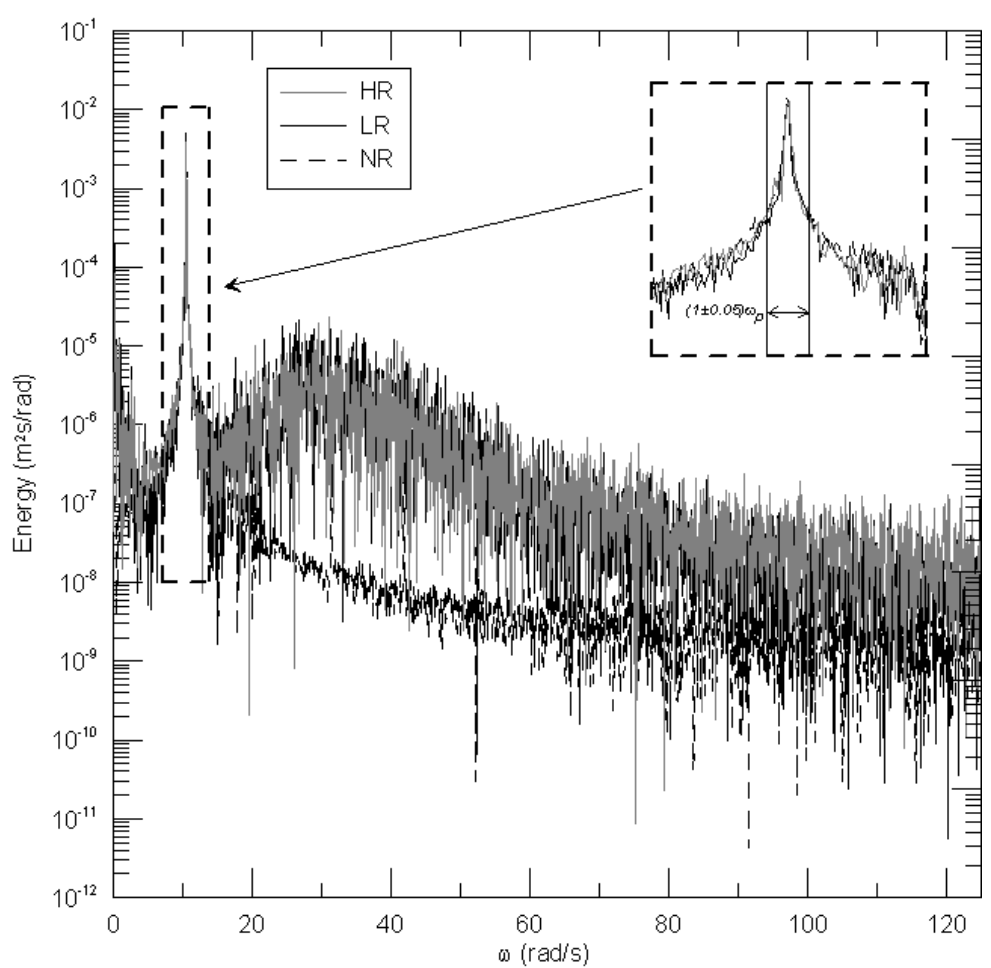

Figure 4. A set of representative wave spectra for the test case $\omega=10.46 \mathrm{rad} \mathrm{s}^{-1}, a k=0.05$ and recorded at a distance of $10.35 \mathrm{~m}$ from the wave generator: No rainfall (NR) dashed line; Low rainfall (LR) solid line; High rainfall (HR) gray line. Note the negligible difference in the spectral wave energy for the LR and HR cases. The inset region shows the frequency region used to characterise local monochromatic wave energy.

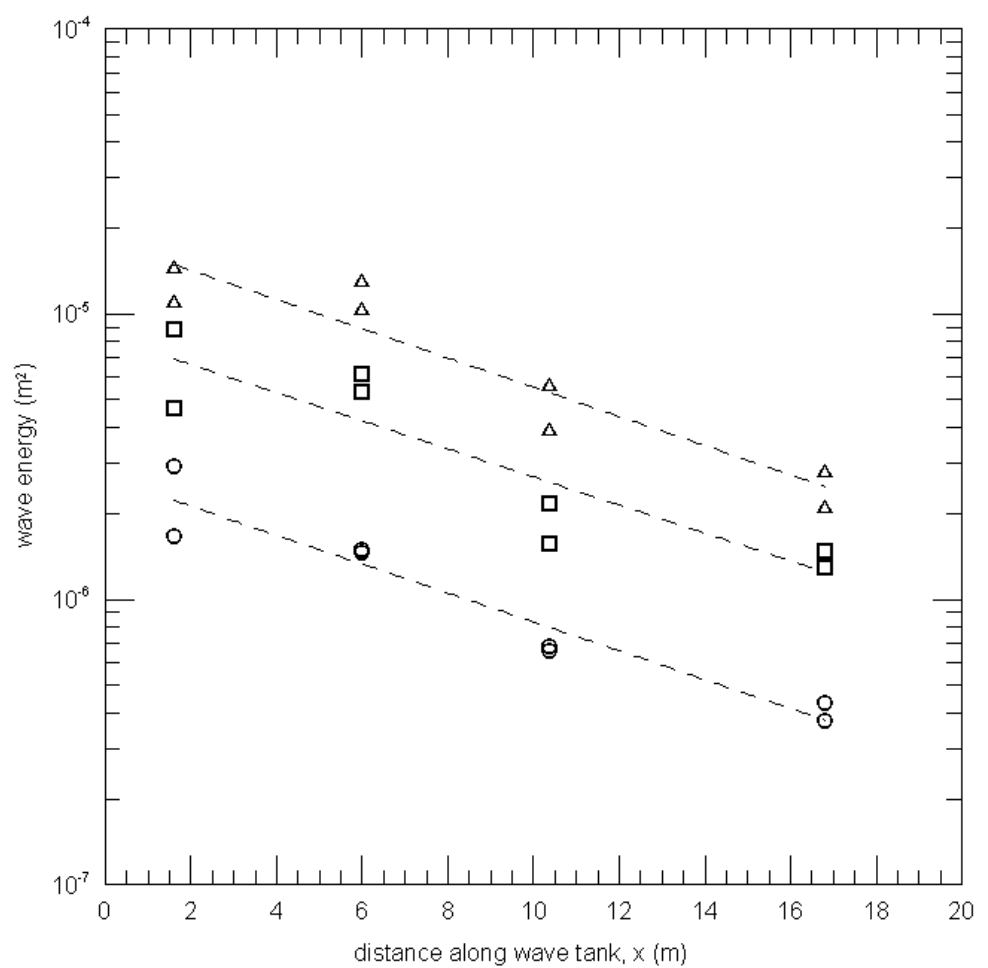

Figure 5. Wave energy as a function of distance from the wavemaker for the highest rainfall condition with $\omega$ $=15.7 \mathrm{rad} \mathrm{s}^{-1}$ : circles $a k=0.05$, squares $a k=0.10$; triangles $a k=0.15$. The dashed lines show the exponential best fits used to determine the attenuation rate. Note the fitted lines are near-parallel, indicating the weak dependency of attenuation on wave steepness. 


\section{RESULTS}

Figure 6 shows the velocity fluctuations obtained from the static ADV measurements. No significant differences were observed between the LR and HR rainfall scenarios. Measured velocity fluctuation levels are very low $\left(<0.003 \mathrm{~m} \mathrm{~s}^{-1}\right)$ and are rapidly decaying with depth. Turbulent velocity fluctuation profiles induced by whitecapping and wind shearing in the ocean are of similar characteristics but of stronger intensity and less steep gradients (Craig and Banner 1994).

Figure 7 shows the $l$ measured profiles for both rainfall scenarios. Note that there are no significant differences between the LR and HR scenarios as well as in Figure 6.

Due to the ADV geometry no measurements were taken at lower depths. However a value of $u^{\prime}=0.0033 \mathrm{~m} \mathrm{~s}^{-1}$ can be obtained when the velocity fluctuations profile is extrapolated to the roughness length $z_{0}=0.023 \mathrm{~m}$ obtained from fitting Eq. (3) to the measurements in Figure 7.

$$
l=\kappa\left(|z|+z_{0}\right)
$$

where $l=$ integral turbulence length scale, $\kappa=0.41$ Von Karman constant, $z=$ vertical coordinate, $z_{0}=$ roughness length (Craig and Banner 1994).

Figure 8 shows measured total wave attenuation coefficient $\left(\Delta_{T}\right)$ obtained for all rainfall scenarios. Note the two rainfall rates producing similar wave attenuation.

The rainfall-turbulence wave attenuation coefficient $\left(\Delta_{R}\right)$ plotted in Figure 9 was calculated from Eq. (4) assuming that the measured viscous attenuation coefficient $\left(\Delta_{\text {visc }}\right)$ can be subtracted from the total attenuation.

$$
\Delta_{T}=\Delta_{\text {visc }}+\Delta_{R}
$$

where: $\Delta_{T=}$ total wave attenuation coefficient (measured LR and HR in Figure 8), $\Delta_{\text {visc }}=$ viscous wave attenuation coefficient (NR in Figure 8), $\Delta_{R}=$ rainfall generated turbulence wave attenuation coefficient.

Measured wave attenuation and velocity fluctuations due to rainfall generated turbulence are compared to existing wave turbulence interaction theories.

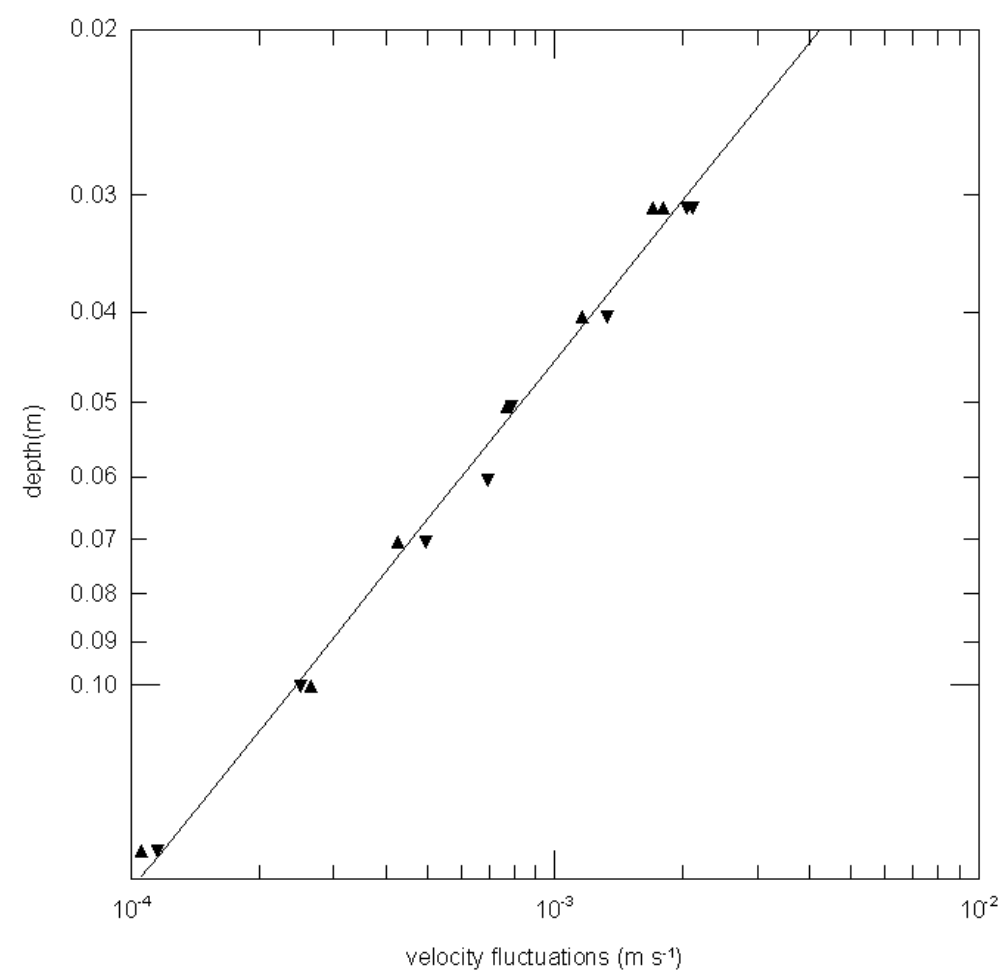

Figure 6. Vertical profiles of velocity fluctuations obtained from the static ADV measurements for both rainfall conditions during this investigation. LR and HR conditions are indicated by downward and upward pointing triangles respectively. The best power fit $u^{\prime}=4.14 z^{-1.77}$ is shown as a solid line. 


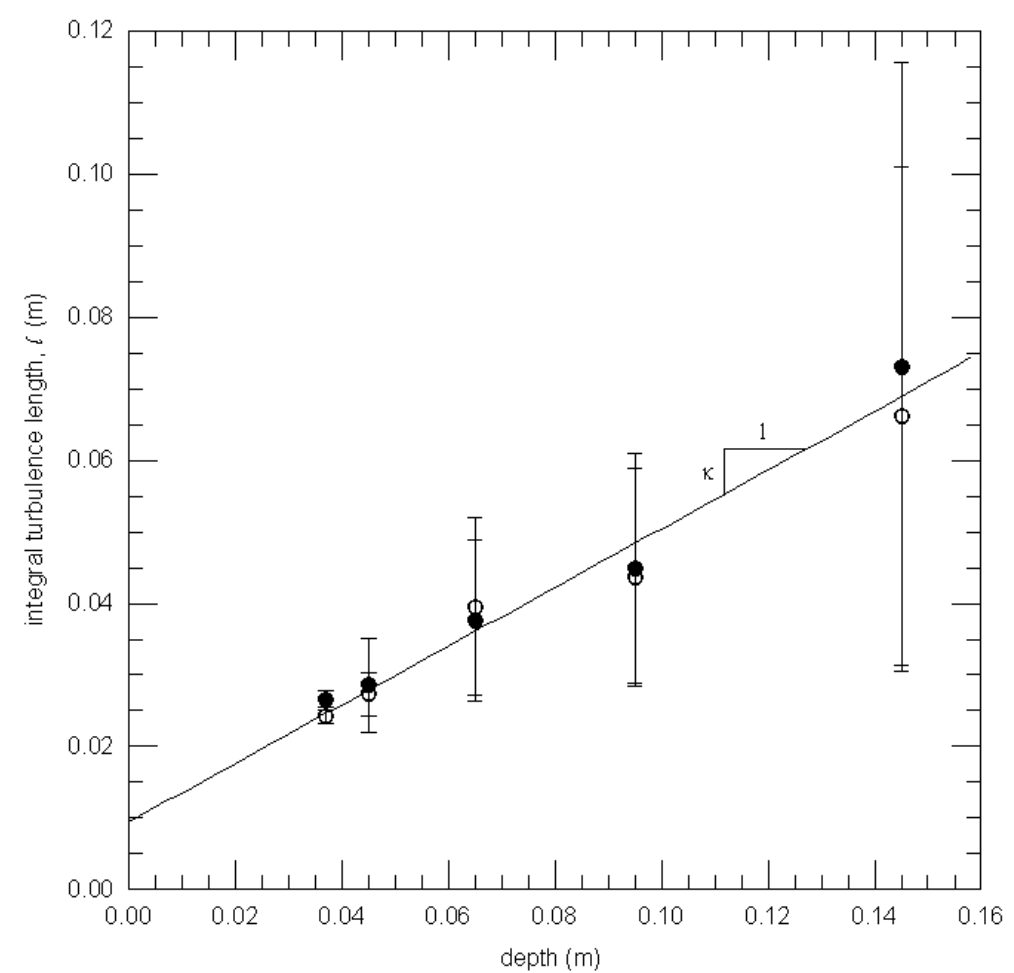

Figure 7. Vertical profile of the turbulent integral length (I) obtained from the turbulence wave number spectra (ADV mounted on a moving trolley measurements). The dashed line shows a linear fit with a slope equal to the Von Kármán parameter, $K=0.41$.

\section{Teixeira and Belcher (2002) model}

Teixeira and Belcher (2002) developed a rapid distortion model to study the interaction of a single irrotational monochromatic wave and weak turbulence. The model is applicable when the orbital velocity is larger than the turbulence and the slope of the wave is sufficiently high that the straining of the turbulence by waves dominates over the straining of the turbulence itself. The model provides a direct estimate of the wave attenuation coefficient which, for deepwater finite amplitude is an increasing function of the square of the velocity fluctuations and the fourth power of the wave frequency.

$$
\Delta_{R}=4 \frac{\alpha \cdot u^{\prime 2} \omega^{4}}{g^{3}}
$$

Where: $\Delta_{R}=$ wave attenuation coefficient due to rainfall, $\alpha=0.6$ constant, $u$ '= velocity fluctuations at the depth of the turbulence boundary layer, $g=$ gravity acceleration, $\omega=$ wave frequency.

\section{Boyev (1971) model}

Boyev (1971) proposed a model for the attenuation of low amplitude deepwater surface waves by intense turbulence. The model assumes that the energy contained in the turbulence is much greater than the energy contained by the waves and that the mechanism responsible for the wave attenuation is the interaction between vertical mixing caused by the turbulent motion and the non-uniformity of the wave flux over the depth. Boyev (1971) found that the attenuation coefficient is an increasing function of the spatial spectrum of the velocity fluctuations and the wave frequency given by:

$$
\Delta_{R}=k u^{\prime} \int_{0}^{\infty} \hat{\Phi}_{s}^{1 / 2} s^{-1 / 2}\left(1-e^{-2 \pi \cdot k / s}\right) d s
$$

where: $\Delta_{\mathrm{R}}=$ wave attenuation coefficient, $u^{\prime}=$ turbulent velocity component fluctuation, $k=$ wave number, $\hat{\Phi}_{s}=\Phi_{s} / u^{\prime 2}=$ wave number spectra normalized by the velocity fluctuation, $s=$ turbulence wave number. 


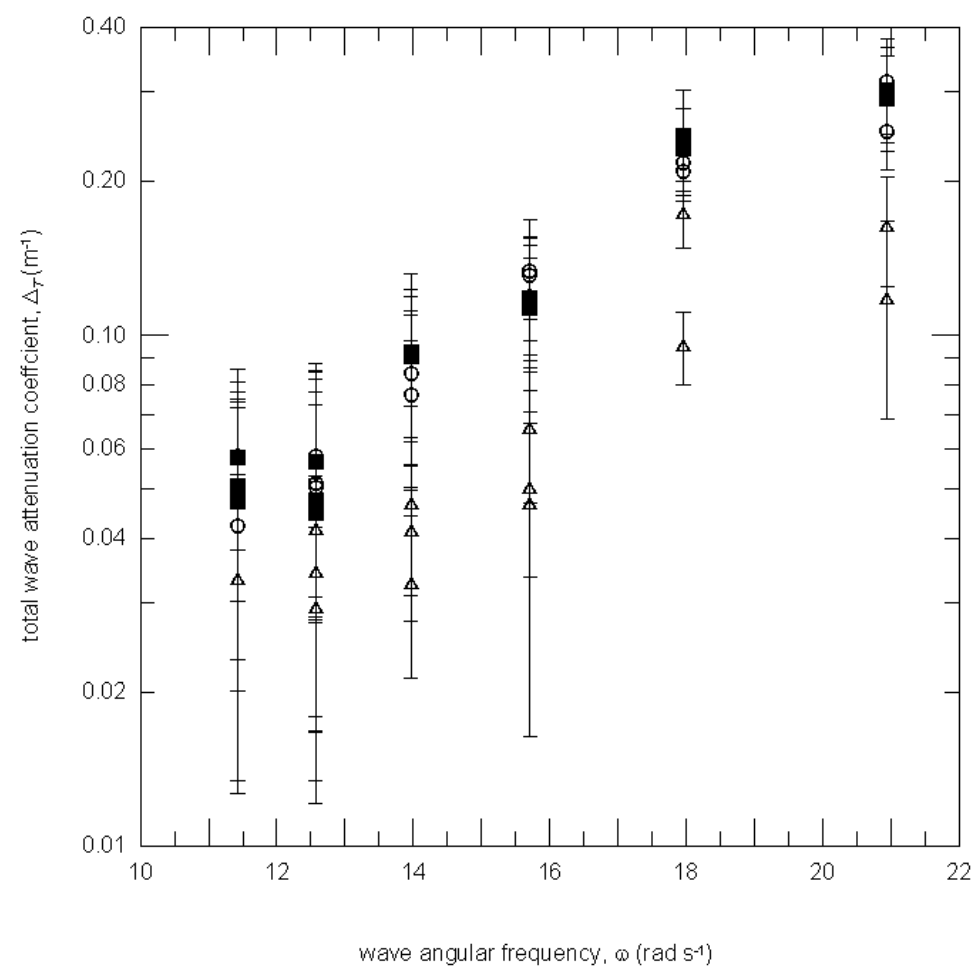

Figure 8. Total wave attenuation coefficient $\Delta_{T}$ as a function of wave angular frequency for different rainfall scenarios. (NR) triangles, (LR) circles, (HR) squares. Statistical errors obtained from correlation coefficients in the exponential fit (Acton 1959, p24).

\section{Comparison with models}

The two models require constant values to represent assumed turbulence fields which are conceptually different to the conditions observed in the ocean, (Figure 6, Craig and Banner 1994).

Comparison with Teixeira and Belcher (2002) was carried out using the extrapolated value of $u^{\prime}=0.0033$ at $z_{0}$ obtained previously. The use of this value requires increasing the original constant $\alpha$ in a factor of 35 in order to match the observations. Figure 9 shows the observed wave attenuation coefficient contrasted with the Teixeira and Belcher (2002) model with the increased $\alpha$ constant.

Boyev (1971) expression is more complicated. However simplifying the turbulence spectrum to the form of Eq. (1) and assuming that the value of the exponential in Eq. (6) is negligible when $k>>s$, the attenuation coefficient is approximately proportional to $\omega^{2}$. Figure 9 also shows a best fitted $\omega^{2}$ curve representing the Boyev (1971) model. 


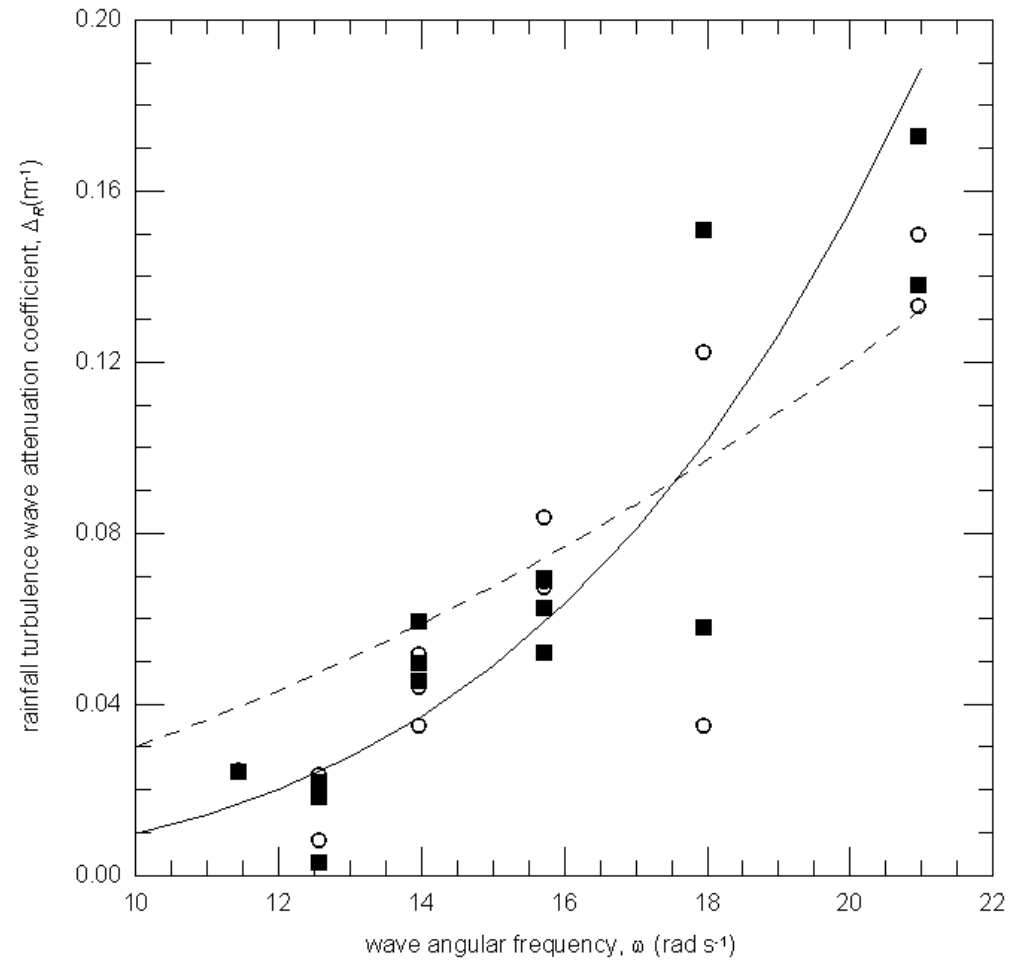

Figure 9. Observed wave attenuation coefficient due to rainfall compared to theoretical estimates. LR (circles), HR (squares), Teixeira and Belcher (2002) (solid line) and a simplification of Boyev (1971) proportional to $\omega^{2}$ (dashed line).

\section{CONCLUSIONS}

We carried out careful measurements of wave attenuation caused by surface generated turbulence. Observations agree, in a broad sense, with theoretical estimate of Teixeira and Belcher (2002).

Simulated rainfall is spatially and temporarily homogeneous producing turbulence at the surface without generating a net horizontal momentum. The rainfall generated turbulence profile decays rapidly with depth in a similar way to that formed under whitecapping and wind shear conditions in the ocean (Craig and Banner 1994). However turbulence intensities induced by simulated rainfall are very weak and not strongly dependent on rainfall intensity. This is an inconvenience if a broad range of turbulence intensities needs to be tested.

Theoretical parameterizations of the wave attenuation coefficient are very sensitive to turbulence intensities. This added to the complexity in the measurement of turbulence and its estimate from the forcing parameters introduces significant challenges to the applicability of such parameterizations in wave modelling.

Moreover models require a turbulence intensity value representative from a depth decaying profile. Teixeira and Belcher (2002) recommend the use of $u$ ' measured at a depth equal to the integral length scale $(l)$. However their rapid distortion turbulence theory assumes a turbulence profile based on Hunt and Graham (1978) where grid generated turbulence is convected by a free stream and blocked by a solid boundary moving at the same speed of the mean flow. This is conceptually a different process to what occurs in the ocean where turbulence is generated at the surface and diffused down the water column (Craig and Banner 1994).

Only recently source-sink terms have been explicitly included in wave propagation models (Tolman 2009). Conventionally turbulence has been implicitly included in the generation and dissipation terms (wind generation, whitecapping and wave breaking). There is potential for significant improvements in wave propagation models if ocean turbulence can be appropriately characterized and identified from the wave generation and dissipation processes. The inclusion of an explicit turbulence source-sink term shall require modifications in the generation and dissipation terms.

The Teixeira and Belcher (2002) wave attenuation model with appropriate calibration and an adequate characterization of the turbulence profile in the ocean, such as the (Craig and Banner 1994) model, could provide significant improvements in wave modelling predictions. Future advances for 
applications in ocean wave modelling should include a re-analysis of the theory with experimental validation using turbulence profiles representative of the conditions observed in the ocean.

\section{ACKNOWLEDGMENTS}

The authors would like to express their appreciation for the technical support provided by the staff of the Water Research Laboratory, especially Mrs. Wendy Thomason-Harper, Mrs. Anna Blacka, Mr. John Hart and Mr. Hamish Studholme, CONICYT - Bicentennial Scholarships - Government of Chile. Michael Banner \& William Peirson gratefully acknowledge support from the Australian Research Council under Discovery Project 0985602 and the UNSW Faculty of Engineering for providing an equipment grant for the construction of the rain generator.

\section{REFERENCES}

Acton, F. S. 1959. Analysis of Straight-Line Data. Wylie.

Ardhuin, F. and A. D. Jenkins. 2006. On the interaction of surface waves and upper ocean turbulence, J. Phys. Oceanogr., 36, pp. 551-557.

Benjamin, T.B. and J.E. Feir. 1967. The disintegration of wavetrains in deep water, Part 1, J. Fluid Mech., 27, pp. 417-430.

Boyev, A. G. 1971. The damping of surface waves by intense turbulence, Izv. Atmos. Ocean Phys. 7, 31-36.

Bradshaw, P. 1971, An introduction to Turbulence and its Measurement, Pergamon Press, First edition.

Craig, P. and M. Banner. 1994. Modelling wave-enhanced turbulence in the ocean surface layer, $J$. Phys. Oceanogr., 24, pp. 2546-2559.

Hunt, J. and J. Graham. 1978. Free-stream turbulence near plane boundaries, J. Fluid Mech. 84, pp. 209-235.

Nikora, V. and D. Goring. 1998. ADV Measurements of Turbulence: Can We improve Their Interpretation?, J. Hydr. Engrg. 124, Issue 6, pp. 630-634. ASCE.

Peirson, W.L., A.W. Garcia and S.E Pells. 2003: Water wave attenuation due to opposing wind, J. Fluid Mech. 487, pp. 345-365.

Pope, S. 2000. Turbulent flows, Cambridge University Press.

Shelton, C.H., R. D. von Bernuth, and S.P. Rajbhandari. 1985. A Continuous Application Rainfall Simulator, American Society of Agricultural Engineers, Vol 28(4), Paper N 84-2049.

Teixeira, M. A. C. and S.E. Belcher. 2002. On the Distortion of Turbulence by a Progressive Surface Wave, J. Fluid Mech. 458, pp. 229-267.

Tennekes, H. and J. L. Lumley. 1974. A First course in Turbulence, $15^{\text {th }}$ printing MIT Press Design Department.

Tolman, H. 2009. User manual and system documentation of WAVEWATCH III ${ }^{\text {TM }}$ version 3.14, Technical note, MMAB Contribution No. 276, NOAA.

Voulgaris, G. and J. Trowbridge. 1998. Evaluation of the acoustic Doppler velocitmeter (ADV) for Turbulence Measurements, Journal of Atmospheric and Ocean Technology, 15, pp. 272-289. American Meteorological Society. 